\title{
Cuidados de enfermagem frente às complicações do cateter central de inserção periférica em neonatos
}

Nursing care in view of complications regarding the peripherally inserted catheter in newborns

Cuidados de enfermería frente a las complicaciones del catéter central de inserción periférica en neonatos

Cátia Aline Silva Swerts ${ }^{1}$, Adriana Olímpia Barbosa Felipe², Karen de Miranda Rocha ${ }^{3}$, Cláudia Umbelina Baptista Andrade ${ }^{4}$

\footnotetext{
${ }^{1}$ Enfermeira, Mestre em Saúde. Professora da Universidade José do Rosário Vellano (UNIFENAS). Alfenas, MG, Brasil. E-mail: catia.silva@unifenas.br.

2 Enfermeira, Mestre em Saúde. Professora da UNIFENAS. Alfenas, MG, Brasil. E-mail: adrianaofelipe@yahoo.com.br.

${ }^{3}$ Enfermeira. Alfenas, MG, Brasil. E-mail: karen.enfermagem@hotmail.com.

${ }^{4}$ Enfermeira, Mestre em Ciências da Saúde. Professora da UNIFENAS. Alfenas, MG, Brasil. E-mail: claudia.andrade@unifenas.br.
}

\section{RESUMO}

Este estudo objetivou avaliar os cuidados de enfermagem frente às complicações relacionadas ao cateter central de inserção periférica (CCIP) em neonatos. Trata-se de pesquisa descritiva, observacional com três enfermeiras e vinte técnicas de enfermagem, utilizando-se de instrumento estruturado para registro das observações e complicações do CCIP. As enfermeiras demonstraram capacidade técnica para uso do CCIP e 75\% das técnicas de enfermagem participaram de educação continuada. A veia cefálica foi escolhida pelas enfermeiras como local de primeira escolha do CCIP, seguida da veia basílica. Diante da obstrução do cateter é utilizada a técnica das duas seringas conectadas na torneira de três vias. Os resultados deste estudo oferecem subsídios para que profissionais de enfermagem fiquem atentos para intervenções necessárias frente às complicações do uso do CCIP, bem como chamam a atenção para a importância de prover cursos de qualificação que fundamentem boas práticas dos cuidados de enfermagem.

Descritores: Cateterismo Venoso Central; Recém-Nascido; Equipe de Enfermagem; Unidades de Terapia Intensiva Neonatal.

\section{ABSTRACT}

The objective of this study was to evaluate the nursing care in view of complications related to peripherally inserted central catheters (PICC) in newborns. This descriptive, observational study was conducted with three nurses and twenty nursing technicians, using a structured instrument to record PICC-related observations and complications. The nurses showed having the technical skills to use the PICC and $75 \%$ of the nursing technicians attended continuing education. Nurses stated the cephalic vein as the first-choice location to place the PICC, followed by the basilica vein. In case the catheter becomes obstructed, this is solved using the technique of two syringes connected to a 3-way stopcock. The present study results offer support for nursing professionals to become aware regarding the necessary interventions in view of complications involving the use of the PICC, and also highlight the importance of offering training courses that promote foundations of good nursing care practices.

Descriptors: Catheterization, Central Venous; Infant, Newborn; Nursing, Team; Intensive Care Units, Neonatal.

\section{RESUMEN}

El estudio objetivó evaluar los cuidados de enfermería para enfrentar complicaciones relativas al catéter central de inserción periférica (CCIP) en neonatos. Investigación descriptiva, observacional, con tres enfermeras y veinte técnicas de enfermería. Se utilizó instrumento estructurado para registro de observaciones y complicaciones del CCIP. Las enfermeras demostraron capacidad técnica para utilizar el CCIP; 75\% de las técnicas de enfermería participaron de capacitación permanente. El sitio de punción de primera elección el CCIP para las enfermeras fue la vena cefálica, seguida de la vena basílica. Ante la obstrucción del catéter, se utiliza la técnica de las dos jeringas conectadas a la canilla de tres vías. Los resultados de este estudio indican que los profesionales de enfermería deben permanecer atentos a intervenciones necesarias frente a complicaciones de uso del CCIP, y llaman la atención acerca de la importancia de ofrecer cursos de calificación que fortalezcan las buenas prácticas de cuidados de enfermería.

Descriptores: Cateterismo Venoso Central; Recién Nacido; Grupo de Enfermería; Unidades de Cuidado Intensivo Neonatal. 


\section{INTRODUÇÃO}

Nas últimas décadas, os acessos venosos centrais estão sendo mais indicados nas Unidades de Terapias Intensivas para salvar a vida dos clientes que necessitam de acesso venoso de longa duração(1). O avanço tecnológico tem acrescentado para o aumento da sobrevida dos neonatos. Um acesso vascular com maior tempo de durabilidade é essencial para a sobrevida desse cliente $^{(2)}$. O cateter central com longa permanência permite uma confiabilidade de acesso às grandes veias centrais para infusões de líquidos, derivados de sangue, administração de medicações e soluções com altos níveis de $\mathrm{PH}$ e osmolaridade $\mathrm{e}^{(3-4)}$.

O Cateter Central de Inserção Periférica (CCIP) é um dispositivo inserido em uma veia superficial da extremidade do corpo e progride, por meio de uma agulha introdutora, até o terço médio da veia cava superior ou veia cava inferior ${ }^{(5)}$.

Nas unidades de cuidados intensivos neonatais a utilização do CCIP tem se tornado um exercício frequente, sendo os enfermeiros os profissionais responsáveis por sua inserção, manutenção e remoção. Assim, cada vez mais, os enfermeiros buscam capacitar-se para essa prática. No Brasil, a atribuição e competência ética legal para sua execução é definida na Resolução n. ${ }^{\circ}$ 258/2001 do Conselho Federal de Enfermagem ${ }^{(6-7)}$.

Esse tipo de cateter tem sido relatado com baixos níveis de infecções e de complicações tanto no ato da inserção, como durante sua manutenção e remoção(8).

Apesar dos inúmeros benefícios atribuídos ao CCIP, os profissionais devem estar atentos aos riscos relacionados ao seu uso, associados a algumas complicações que podem ocorrer na inserção, durante a manutenção e a remoção. Tais complicações ocorrem por problemas mecânicos como obstrução, ruptura do cateter, perfuração do vaso, extravasamento, trombose, problemas infecciosos, sepse relacionada ao cateter, hematoma, posição inadequada do cateter e pneumotórax ${ }^{(5,7)}$.

Os procedimentos de manutenção do CCIP mostram diversidade entre a prática assistencial e o protocolo institucional, sendo responsáveis pela ocorrência de algumas complicações ${ }^{(9)}$. Neste contexto, o estudo teve como objetivo avaliar os cuidados de enfermagem frente às complicações relacionadas ao CCIP em neonatos. A melhoria da qualidade da assistência de enfermagem visa reduzir a morbidade e mortalidade neonatal em decorrência às complicações inerentes ao uso do CCIP.

\section{METODOLOGIA}

Estudo descritivo observacional com abordagem quantitativa. O estudo foi previamente aprovado pelo Comitê de Ética em Pesquisa da Universidade José do Rosário Vellano (UNIFENAS), sob o Parecer n. ${ }^{\circ}$ 21/2010 e realizado em conformidade com a Resolução do Conselho Nacional de Saúde n. ${ }^{\circ}$ 196/96, que trata de pesquisa envolvendo seres humanos ${ }^{(10)}$.

O estudo foi realizado no período de maio a julho de 2010 na Unidade de Terapia Intensiva Neonatal (UTIN) de um Hospital Universitário no Sul de Minas Gerais. A unidade é composta por 12 leitos (seis intensivos e seis semi-intensivos) com 24 profissionais de enfermagem (quatro enfermeiros e 20 técnicos de enfermagem).

A amostra de conveniência do estudo foi composta por três enfermeiros ( $75 \%$ da população total) e 20 técnicos em enfermagem (100\% da população total) atuantes na UTIN, nos turnos matutino, vespertino e noturno. Houve a exclusão apenas de uma enfermeira por questões éticas, em função de ser a pesquisadora responsável deste estudo.

No primeiro momento, foi realizado um estudo observacional com a utilização de um instrumento previamente estruturado para o registro das observações. Desse instrumento, constaram dados de identificação e cuidados executados pela equipe de enfermagem frente às complicações com o cateter, no período de um mês, sendo uma semana em cada turno de trabalho durante duas horas, perfazendo um total de 56 horas e quatro semanas determinadas pela rotina de trabalho dos profissionais em cada turno de atividade: matutino ( $8 \mathrm{~h}$ às $10 \mathrm{~h}$ ), vespertino (14 às $16 \mathrm{~h}$ ) e noturno (20h às 22h).

Após o período de observação, foi aplicado à equipe de enfermagem um questionário estruturado do qual constaram dados de identificação e clínicos do recémnascido (data de nascimento, sexo, peso, diagnóstico, tempo de internação, identificação para uso do CCIP, procedência e número do prontuário), cuidados executados pela equipe de enfermagem frente às complicações relacionadas ao cateter (flebite, trombose, infiltração, extravasamento, infecção local, complicações sistêmicas, embolia gasosa e embolia por cateter). Os 
dados do questionário foram comparados com aqueles obtidos no período de observação.

Após o encerramento da coleta de dados, os dados foram analisados e tabulados em frequências absolutas e percentuais.

\section{RESULTADOS}

Vinte (87\%) participantes eram técnicos em enfermagem e três (13\%) eram enfermeiros; todos do sexo feminino. Em relação ao período trabalhado, 12 (52,2\%) profissionais trabalhavam no período noturno e $11(47,8 \%)$ atuavam no setor há mais de três anos.

A maioria das enfermeiras e técnicas de enfermagem $(69,5 \%)$ responderam ser importante o registro de todas as informações sobre o cateter, desde o termo de consentimento assinado pelos responsáveis legais, procedimento de inserção detalhado, cuidados diários, complicações, até a remoção do mesmo. Por outro lado, $21,8 \%$ dos profissionais de enfermagem consideram importante o registro apenas dos dados referentes à inserção do cateter, e para $8,7 \%$ dos participantes do estudo consideraram importante somente o registro diário das intercorrências e complicações relacionadas ao uso do cateter.
Na UTIN, observou-se a existência de uma ficha de anotações com os dados de inserção (local de punção, circunferência do membro em centímetros, tamanho do cateter em centímetros, comprimento do cateter introduzido em centímetros, degermação, antissepsia do local de inserção, número de punções, localização do cateter, presença de intercorrências - sangramento, edema local, hematoma - data, hora e assinatura do profissional médico/enfermeiro), manutenção (troca do curativo e avaliação da permeabilidade do cateter e condições locais), complicações (flebite, trombose, infiltração, extravasamento, infecção local, complicações sistêmicas, embolia gasosa e embolia por cateter) e remoção do cateter (motivo da retirada, comprimento do cateter e submissão da ponta do cateter para análise microbiológica), que é de responsabilidade do enfermeiro. Os relatórios dos técnicos de enfermagem são preenchidos eletronicamente e deles não constam os dados sobre o cateter.

Quanto à seleção da veia apropriada para inserção do CCIP, duas $(66,6 \%)$ enfermeiras citaram a veia cefálica como ideal para a primeira escolha e, uma enfermeira, $(33,3 \%)$ a veia basílica (Tabela 1$)$.

Tabela 1: Locais selecionados pelos enfermeiros para acesso venoso periférico para inserção do Cateter Central de Inserção Periférica (CCIP). Sul de Minas Gerais, MG, 2010.

\begin{tabular}{cc}
\hline Veias & $\mathbf{f}(\%)$ \\
\hline Auricular Anterior & 0 \\
Auricular Posterior & Axilar \\
Basílica & 0 \\
Braquial & $1(33,3)$ \\
Cefálica & 0 \\
Cubital & $2(66,6)$ \\
Jugular externa & 0 \\
Safena & 0 \\
Temporal & 0 \\
\hline
\end{tabular}

Diante das implantações de CCIP observadas, verificou-se que a escolha não está pautada nas bases científicas/anatômicas, mas, sim, na facilidade de punção naquele momento.

Diante do surgimento de sinais flogísticos na inserção do CCIP, observou-se que 20 (100\%) das técnicas de enfermagem comunicam à enfermeira do setor e aplicam compressas mornas no local, observando o edema e hiperemia por 24 horas e, se necessário, a enfermeira interrompe a infusão e comunica ao médico plantonista. Tais observações foram confirmadas pelos dados quantitativos colhidos no questionário aplicado.

Frente à obstrução do cateter, todas as enfermeiras afirmaram utilizar a técnica das duas seringas, em que se conecta na extremidade do cateter uma torneira de três vias, por sua vez conectada a uma seringa de $3 \mathrm{~mL}$ com uma solução de desobstrução $(0,01 \mathrm{~mL}$ de heparina e 0,99 $\mathrm{mL}$ de solução fisiológica à $0,9 \%$ ) e a uma seringa de 10 
$\mathrm{mL}$ vazia. No entanto, durante o período de observação, foi constatado que algumas enfermeiras utilizam a seringa de $1 \mathrm{~mL}$ e a de $10 \mathrm{~mL}$ conectada na torneira de três vias.

A Tabela 2 mostra a atitude das enfermeiras quando questionadas em relação às complicações do CCIP. Duas enfermeiras (66,6\%) afirmaram suspender a infusão no cateter e uma (33,3\%) afirmou solicitar exames radiográficos. Observou-se que não existe um protocolo com condutas de enfermagem frente às complicações do CCIP.

Tabela 2: Distribuição, em percentual e número, das atitudes dos enfermeiros quanto às complicações com o Cateter Central de Inserção Periférica (CCIP). Sul de Minas Gerais, MG, 2010.

\begin{tabular}{cc}
\hline Ações dos enfermeiros & $\mathbf{f}(\%)$ \\
\hline Retirada imediata do cateter & 0 \\
Indicação de exame radiográfico & $1(33,3)$ \\
Suspensão da infusão no cateter & $2(66,6)$ \\
Investigação da causa & 0 \\
\hline
\end{tabular}

Quanto ao treinamento recebido para realização da manutenção e cuidados com o cateter, 15 (75\%) técnicas em enfermagem afirmaram participar de educação continuada permanente e, cinco (25\%) relataram receber apenas instruções no próprio setor durante a oferta dos cuidados com o neonato pela supervisora. Todas as enfermeiras afirmaram ser capacitadas para inserção, manutenção e remoção do CCIP.

\section{DISCUSSÃO}

O CCIP é um avanço nas Unidades de Terapia Intensiva Neonatal, no entanto é necessário que a equipe de enfermagem esteja preparada e treinada para prestar assistência de qualidade após sua inserção.

Para que a equipe de enfermagem obtenha o sucesso com a implantação do cateter, esta deve ter conhecimento sobre os riscos envolvidos no seu uso. As complicações durante a sua inserção, manutenção e remoção, são os principais motivos para a retirada prematura do cateter. Os cuidados de enfermagem são fatores essenciais na manutenção do CCIP. A identificação das possíveis complicações relacionadas ao seu uso tornase uma necessidade para esses profissionais que atuam diretamente na sua manipulação.

Em relação ao registro sobre o CCIP, a maioria dos profissionais considerou importante a anotação de todas as informações relacionadas ao cateter, e observou-se a existência de uma ficha de anotações de responsabilidade do enfermeiro. No entanto, não constam informações sobre o cateter no relatório eletrônico dos técnicos de enfermagem, que somente checam nas prescrições médicas as realizações dos flushings de acordo com o prescrito. O protocolo de instalação do CCIP deve conter informações acerca das características do cateter escolhido (diâmetro e volume interno do cateter), do sítio de inserção, de intercorrências durante o procedimento, laudo da radiografia. Além disso, informações relacionadas a manutenção (troca de curativo, avaliação das condições locais e permeabilidade do cateter) e remoção (motivo da retirada, comprimento do cateter retirado e envio da ponta para análise microbiológica) devem ser registradas através de instrumentos impressos ou eletrônicos, o que auxilia a assistência e padroniza o cuidado, elevando, assim, a qualidade da assistência prestada ${ }^{(4,11)}$.

A maior parte das enfermeiras citou a veia cefálica como ideal para a primeira escolha, diferentemente dos achados da literatura que recomendam, como primeira escolha, a veia basílica, pois sua opção se dá pelas características anatômicas favoráveis, como maior calibre, menor número de válvulas, além da localização que facilita a manipulação para a troca dos curativos. Já a veia cefálica foi citada como a segunda opção(12-15). Por outro lado, há estudos que indicam a veia cefálica por esta apresentar uma anatomia favorável e facilitar a troca do curativo $^{(13)}$.

Consideramos relevante a escolha do membro superior direito para inserções do CCIP, devido à maior facilidade de progressão e centralização do mesmo.

A busca de outros locais para inserção do CCIP é decorrente da fragilidade da rede venosa dos neonatos graves com longo período de internação nas unidades de terapia intensivas neonatais ${ }^{(13)}$. Tal fator pode justificar a observação feita na unidade de estudo onde a escolha da 
veia não está pautada nas bases científicas/anatômicas, mas, sim, na facilidade de punção naquele momento.

Diante ao aparecimento de sinais flogísticos, as enfermeiras colocam compressas mornas no local e observam o local por 24 horas e, quando necessário, a enfermeira interrompe a infusão e comunica ao médico plantonista. Outro estudo apresenta as mesmas recomendações diante da identificação de sinais flogísticos $^{(16)}$. Um dos principais sinais indicativos de infecção local, principalmente nas primeiras 24 horas após a inserção do cateter, é o edema do membro, que também pode ocorrer no neonato por hipoalbuminemia(17). Desta forma, a equipe multidisciplinar deve estar atenta, impedindo a retirada precoce do cateter inadvertidamente por confundir o edema com infiltração no membro ${ }^{(15,18)}$.

Todas as enfermeiras utilizam técnica das duas seringas para a desobstrução do cateter, porém foi relatada a utilização da seringa de 3 e $10 \mathrm{~mL}$ conectada a uma torneira de três vias. Em nossa observação, foi constatado que algumas enfermeiras utilizam a seringa de 1 e $10 \mathrm{~mL}$ conectada na torneira de três vias. O cateter é feito de material frágil e de lúmen reduzido, um fator que o torna um cateter de manutenção delicada, pois obstrui com facilidade e rompe facilmente em tentativas de desobstrução. Na unidade, a técnica de desobstrução é amplamente utilizada, porém não há um protocolo de rotina para sua aplicação(18).

A obstrução do cateter pode ser causada por formação de trombos, má posição de sua ponta contra a parede da veia, por dobra ou pinçamento por estruturas anatômicas. A trombose é comum após administração de hemotransfusão e coleta de sangue. Essas manipulações devem ser acompanhadas de irrigações; já as obstruções provocadas por mau posicionamento do cateter na parede da veia, devem ser corrigidas por reposicionamento do cateter ou do próprio paciente. A aspiração do cateter faz com que a ponta funcione como uma ventosa na parede da veia, impedindo a saída de sangue pelo cateter ${ }^{(5)}$.

Quando as enfermeiras foram questionadas quanto a sua atitude diante das complicações do CCIP, 66,6\% delas afirmaram suspender a infusão no cateter e $33,3 \%$ afirmaram solicitar exames radiográficos. Os profissionais sentem dificuldades em investigar as causas das complicações, mesmo diante da vivência desses problemas em seu cotidiano de trabalho. Em caso de suspeita de tracionamento, deve-se proceder à confirmação do posicionamento da ponta do dispositivo por meio de exames radiográficos ${ }^{(5,16)}$.

Todas as enfermeiras afirmaram ser capacitadas para inserção, manutenção e remoção do cateter central de inserção periférica e a maior parte das técnicas em enfermagem, afirmaram participar de educação continuada permanente. A educação continuada estava em fase inicial de implantação, justificando-se uma parcela da equipe não ter recebido treinamento específico quanto aos cuidados com o CCIP.

O enfermeiro deve estar habilitado tanto para inserção como pela manutenção do CCIP, ficando responsável pela prevenção de suas complicações ${ }^{(19)}$.

A educação permanente em saúde implica uma estratégia imprescindível às transformações do trabalho no setor, tornando-o um lugar de atuação crítica, reflexiva, com novas propostas, com uma equipe compromissada e tecnicamente competente, devendo unir a teoria e a prática, identificar e analisar problemas desenvolvendo propostas de mudança ${ }^{(20)}$. Representa para o profissional de saúde oportunidade de autodesenvolvimento, motivação e autorrealização. Por isso há a necessidade de um programa de capacitação dos enfermeiros para adotar a educação permanente, pois esta sustenta o crescimento da equipe fundamental na determinação da qualidade do cuidado e oferece ajuda profissional, fortalecendo o processo de crescimento pessoal e de transformação no âmbito profissional(21).

Por fim, o estudo contou com a participação de aproximadamente $95,8 \%$ da amostra $(n=23)$ de uma Unidade de Terapia Intensiva Neonatal, alvo deste estudo; e mesmo com uma amostra limitada de profissionais e em curto período de tempo, devido ao objetivo do estudo, pode-se observar que os profissionais devem estar atentos e capacitados às complicações que porventura surjam frente à manipulação do CCIP. Tais indicadores sugerem um aprofundamento e detalhamento a partir de novos estudos uma vez que a CCIP é indicado em neonatos.

\section{CONCLUSÕES}

A avaliação dos cuidados frente às complicações do CCIP proporcionou o conhecimento sobre a realidade do uso desse dispositivo em uma Unidade de Terapia 
Neonatal, principalmente por se constatar que a organização do registro de enfermagem apresentou-se insuficiente pelos dados colhidos, apesar da equipe reconhecer a importância do registro de enfermagem acerca do cateter.

Por observação, a equipe de enfermagem da Unidade de Terapia Intensiva Neonatal atua sem padronização das técnicas de manipulação do cateter não priorizando as bases científicas e anatômicas.

Nem toda a equipe de enfermagem está preparada para a manipulação do cateter, pois, os cuidados realizados frente às complicações do CCIP são diferentes entre os enfermeiros, entretanto, para obter o sucesso dessa prática é necessário que os profissionais busquem o conhecimento técnico e científico por meio de educação continuada permanente, e de treinamentos que visam evitar as complicações. De igual forma é fundamental que as intervenções de enfermagem realizadas em problemas já instalados no cateter garantam a sua utilização até o término do tratamento.

Os resultados deste estudo oferecem subsídios para que profissionais de enfermagem fiquem atentos para as intervenções necessárias frente às complicações do cateter central de inserção periférica em neonatos, bem como chamam a atenção para a importância de se prover cursos de qualificação que fundamentem boas práticas dos cuidados de enfermagem.

central de inserción periférica (PICC) en los recién nacidos. Enferm. glob. [Internet]. 2011 [acesso em: 30 mar 2013];10(24). Disponível em: http://dx.doi.org/10.4321/S169561412011000400001.

13. Baggio MA, Bazzi FCS, Bilibio CAC. Cateter central de inserção periférica: descrição da utilização em UTI Neonatal e Pediátrica. Rev Gaucha Enferm [Internet]. 2010 [acesso em: 30 mar 2013];31(1):70-6. Disponível em:

http://dx.doi.org/10.1590/S1983-14472010000100010.

14. Lourenço SA, Ohara CVS. Nurses' Knowledge about the Insertion Procedure for Peripherally Inserted Central Catheters in Newborns. Rev Lat Am Enfermagem [Internet]. 2010 [acesso em: 30 mar 2013];18(2):189-95. Disponível em:

http://dx.doi.org/10.1590/S0104-11692010000200008. 15. Rodrigues ZS, Chaves EMC, Cardoso MVLML. Atuação do enfermeiro no cuidado com o cateter central de inserção periférica no recém-nascido. Rev Bras Enferm [Internet]. 2006 [acesso em: 30 mar 2013];59(5):626-9. Disponível em: http://dx.doi.org/10.1590/S0034-71672006000500006. 16. Phillips LD. Manual de terapia intravenosa. $2^{a}$ ed. Porto Alegre: Artmed; 2001.

17. Pazzini LT. Caracterização genotípica de microrganismos isolados de infecções da corrente sanguínea relacionadas a cateteres em recém-nascidos [monografia]. São Paulo: Unesp; 2010.

18. Gaíva MAM, Scochi CGS. Processo de trabalho em saúde e enfermagem em UTI neonatal. Rev Lat Am Enfermagem [Internet]. 2004 [acesso em: 30 mar 2013];12(3): 469-76. Disponível em: http://dx.doi.org/10.1590/S010411692004000300004.

19. Motta PN, Fialho FA, Dias IMAV, Nascimento L. Cateter central de inserção periférica: o papel da Enfermagem na sua utilização em Neonatologia. HU Revista. 2011;37(2):163-8. 20. Nietsche EA, Backes VMS, Ferraz F, Loureiro L, Schmidt SMS, Noal HC. Política de educação continuada institucional: um desafio em construção. Rev. Eletr. Enf. [Internet]. 2009 [acesso em: 30 mar 2013];11(2):341-8. Disponível em:

http://www.fen.ufg.br/revista/v11/n2/v11n2a15.htm 21. Ricaldoni CAC, Sena RR. Permanent education: a tool to think and act in nursing work. Rev Lat Am Enfermagem [Internet]. 2004 [acesso em: 30 mar 2013];14(6):837-42. Disponível em: http://dx.doi.org/10.1590/S010411692006000600002.

Artigo recebido em 19/04/2011. Aprovado para publicação em 19/06/2012. Artigo publicado em 31/03/2013. 12. Montes SF, Teixeira JBA, Barbosa MH, Barichello E. Aparición de complicaciones relacionadas con el uso del catéter venoso 